\title{
Different Ways of Classifying Drums with Reference to Illustrations and Positioning of Drum Illustrations
}

\author{
By Rolf KJellströM
}

Drum types with reference to illustrations

I Without obvious divisions

II Square cross as unifyıng central figure

a) ordinary square cross

b) with three vertical lines

III Drum with one horizontal main line

A Whole line

a) with square cross

b) with other complete vertical line

c) without vertical line

B Line broken in the muddle

C Line not going out to edge

a) with further vertical line

D Whole lines

a) with square cross

IV Drums with two horizontal lines

A Whole lines

a) without connecting vertical line

b) with connecting vertical line

B Broken lines

C One whole line + one broken

a) with square cross or similar

V Drums with four horizontal lines 


\section{POSITION OF DRUM FIGURES}

I Position in relation to signs on drum

1 Horizontal lines

11 Drum division

111 Line 1

112 Line 2

113 Line 3

114 Line 4

12 Other lines

121 Square cross

122 Lines branching out from rhombus

123 Other horizontal lines

$2 \quad$ Vertical lines

21 Lines joining 2 horizontal lines

22 Vertical bar of square cross

23 Other central vertical lines

24 Other lines vertical to the side

25 Vertical lines going out to the edge of the frame

$3 \quad$ Spaces

The various spaces on the drum are numbered differently, so that the horizontal lines are indicated as $\mathrm{B} 1$, no 2 from the top $=\mathrm{B} 2$ etc

The space which is formed by the top part of the drum and the first horizontal line is called U1

Depending on whether it is the right hand or the left hand side, the space is called 1 or 2 The next unit on the left-hand side is 3 and on the opposite side 4 etc Uneven numbers here thus refer to the left hand side

4 Position with reference to the above-mentioned surface or space

41 "on It"

42 near

43 floating freely

44 in relation to the square cross: 
4.4.1. over the centre of the square cross

4.4.2. under the centre of the square cross

4.4.3. all along the square cross

4.4.4. all along the square cross and under it

5. Connection between figures

With one "main" figure and "xx figures". The position is recorded with a figure 1-12 in relation to the main figure (clockwise).

\section{Position without relation to other signs}

Every figure is given a centre of gravity. Its position determines the figure's place on the drum, which is divided into ten percent portions vertically and horizontally. We thus obtain concentric figures. 